\title{
Genotoxicity and Gene Expression in the Rat Lung Tissue Following Instillation and Inhalation of Different Variants of Amorphous Silica Nanomaterials $\left(\mathrm{aSiO}_{2} \mathrm{NMs}\right)$
}

\section{Fátima Brandão}

National Institute of Health Doctor Ricardo Jorge: Instituto Nacional de Saude Doutor Ricardo Jorge

\section{Carla Costa}

National Institute of Health Doctor Ricardo Jorge: Instituto Nacional de Saude Doutor Ricardo Jorge

Maria João Bessa

Institute of Public Health of the University of Porto

\section{Elise Dumortier}

Université de Namur: Universite de Namur

Florence Debacq-Chainiaux

University of Namur: Universite de Namur

\section{Roland Hubaux}

StratiCELL

\section{Michel Salmon}

StratiCELL

\section{Julie Laloy}

University of Namur: Universite de Namur

\section{Miruna S. Stan}

University of Bucharest: Universitatea din Bucuresti

\section{Anca Hermenean}

Vasile Goldis University of Arad Faculty of Medicine: Universitatea de Vest Vasile Goldis din Arad Facultatea de Medicina

\section{Sami Gharbia}

Vasile Goldis University of Arad Faculty of Medicine: Universitatea de Vest Vasile Goldis din Arad Facultatea de Medicina

\section{Anca Dinischiotu}

University of Bucharest: Universitatea din Bucuresti

\section{Anne Bannuscher}

University of Fribourg: Universite de Fribourg

\section{Bryan Hellack}


UBA: Umweltbundesamt

\section{Andrea Haase}

BfR: Bundesinstitut fur Risikobewertung

Sónia Fraga ( $\nabla$ teixeirafraga@hotmail.com )

Instituto Nacional de Saude Doutor Ricardo Jorge IP https://orcid.org/0000-0001-9386-2336

João Paulo Teixeira

National Institute of Health Doctor Ricardo Jorge: Instituto Nacional de Saude Doutor Ricardo Jorge

\section{Keywords:}

Posted Date: October 27th, 2020

DOl: https://doi.org/10.21203/rs.3.rs-96206/v1

License: (a) (i) This work is licensed under a Creative Commons Attribution 4.0 International License. Read Full License

Version of Record: A version of this preprint was published at Nanomaterials on June 7th, 2021. See the published version at https://doi.org/10.3390/nano11061502. 


\section{Abstract}

The authors have requested that this preprint be removed from Research Square. 